\title{
Caracterização de Turfa e Vermiculita Hidrofobizadas por Espectroscopia de Absorção na Região do Infravermelho- FTIR
}

\author{
Dafne Ravena Pascoal de Morais*(Estudante de Engenharia de Petróleo, Departamento de \\ Ciências Ambientais e Tecnológicas, UFERSA); \\ Regina Celia de Oliveira Brasil Delgado(Professor(a), Departamento de Ciências Ambientais e \\ Tecnologicas, UFERSA); \\ André Luís Novais Mota(Professor(a), Departamento de Ciências Ambientais e Tecnologicas, \\ UFERSA); \\ Rodrigo César Santiago(Professor(a), Departamento de Ciências Ambientais e Tecnologicas, \\ UFERSA)
}

Email*: dafnepascoal@yahoo.com.br

resumo:

Materiais naturais como minerais e argilo-minerais tem sido alvo de estudos para o uso alternativo em áreas ambientais com o propósito de solucionar problemas diversos que causam impactos ao meio ambiente. A turfa e a vermiculita vêm ganhando atenção atualmente devido ao poder que apresentam em adsorver impurezas de água e efluentes contaminados com hidrocarbonetos e metais, entre outras substâncias tóxicas. A turfa é classificada como um organo-mineral originado da decomposição de vegetais de pequeno desenvolvimento em ambiente de água doce. É um material poroso e altamente polar. A vermiculita é um mineral oriundo das rochas ígneas, ocorre nas rochas alcalinas e às vezes em pegmatitos e geralmente se forma do intemperismo sofrido pela biotita. Diversos métodos tem sido alvo de estudos visando à ativação ou amento da capacidade desses materiais para serem usados como adsorventes de substâncias poluentes, com destaque para a hidrofobização, que consiste em aumentar a capacidade de adsorção de um material, com a característica de aversão a agua, tornando-se melhor adsorvente. Inúmeros trabalhos atestam a eficiência da cera de carnaúba no processo de hidrofobização de materiais argilosos, aumentando a afinidade a compostos orgânicos dos mesmos. Este trabalho teve como objetivo caracterizar a turfa e a vermiculita na forma natural e hidrofobizada com cera de carnaúba pela técnica de absorção da região do infravermelho por transformada de Fourier, visando conhecer melhor as propriedades desses materiais. Os resultados obtidos nas análises atestaram a heterogeneidade dos materiais estudados como também, comprovaram a aderência da cera de carnaúba utilizada no processo de hidrofobização nas bandas $\mathrm{C}=\mathrm{O}$ e $\mathrm{OH}$ característicos da cera e presente nos materiais. A confirmação da presença de grupos funcionais orgânicos nos adsorventes torna a vermiculita e turfa adsorventes potenciais para remoção de contaminantes através da adsorção.

Palavras-chaves: Argilo-minerais; Adsorvente; Vermiculita; Hidrofobização.

\section{INTRODUÇÃO}

A utilização de materiais adsorvedores de substâncias poluentes de águas e efluentes vêm sendo amplamente considerada atualmente. Materiais como carvão ativado, sílica e alumina apresentam posição de destaque entre os mais utilizados, entretanto, ainda são considerados de custo elevado e, portanto, pesquisas por novos materiais, mais abundantes e 
que apresentem ampla disponibilidade e custos mais baixos tornaram-se importantes (COELHO et al, 2007). As propriedades adsorventes de minerais e argilo-minerais vêm sendo amplamente testadas em várias pesquisas (FRANCHI, 2004) com objetivo de aliar custos baixos com preservação ambiental.

A vermiculita é um silicato hidratado de magnésio, alumínio e ferro com uma estrutura micáceo-lamelar e clivagem basal. Suas propriedades de superfície, somadas aos elevados valores de área superficial específica, porosidade e carga superficial (negativa) fazem da mesma um material adequado para o uso como adsorvente (UGARTE et al., 2005).

A turfa é uma substância fóssil, organo-mineral, originada da decomposição de restos vegetais, encontrada em áreas alagadiças como várzeas de rios, planícies costeiras e regiões lacustres (FRANCHI, 2000; FRANCHI et al, 2003). Sob o ponto de vista físico-químico, é um material poroso, altamente polar, com elevada capacidade de adsorção para metais de transição e moléculas orgânicas polares (FRANCHI et al, 2004).'

As várias ocorrências de turfa e vermiculita encontradas no nordeste do Brasil, aliada aos baixos custos, torna esses materiais interessantes para serem utilizados como adsorventes.

Diversas são as pesquisas (MARTINS, 2000) feitas nos últimos tempos sobre a hidrofobização, e os materiais que possam atuar como agentes hidrofobizantes, ou seja, contribuir para que o adsorvente se torne um material com aversão a água e assim aumentar a capacidade de adsorção sobre os materiais presentes. A cera de carnaúba é composta por hidrocarbonetos, ésteres, ácidos e hidroxiácidos, compostos que proporcionam propriedades de alto poder de hidrofobização em materiais adsorventes como argilas, tornando os materiais com afinidade a compostos orgânicos (BORBA, 2013). Estudos realizados por Curbelo (2002), também, atestam a eficiência da cera de carnaúba no processo de hidrofobização.

Diante desse cenário, este trabalho tem o objetivo de conhecer as características adsorventes da turfa e vermiculita na forma natural e hidrofobizada utilizando a técnica de espectroscopia de absorção da região do infravermelho.

\section{METODOLOGIA}

\subsection{Obtenção dos Materiais}

A vermiculita utilizada neste trabalho é do tipo expandida, e foi doada pela empresa Maia Macedo Impermeabilizantes LTDA localizada na cidade de João PessoaPB e a turfa pela empresa Ecofertil Agropecuária LTDA localizada na cidade de Mossoró-RN.

\subsection{Processo de hidrofobização}

Nesta etapa as amostras de vermiculita expandida e turfa foram submetidas a tratamento com cera de carnaúba, Para cada grama (g) da vermiculita expandida e da turfa, foram adicionados $20 \%$ em peso de cera de carnaúba. A cera de carnaúba foi colocada em um recipiente de alumínio sobre uma chapa aquecedora a temperatura média de $100^{\circ} \mathrm{C}$. O controle da temperatura foi realizado para evitar a evaporação da cera, que ocorre acima de $200^{\circ} \mathrm{C}$. Ao aquecer e derreter a cera, o material adsorvente foi adicionado e misturado por 1 minuto até homogeneizar. Posteriormente, esperou-se o material esfriar até atingir a temperatura ambiente e acondicionou-se os mesmos em recipientes apropriados. 


\subsection{Caracterização dos materiais por Espectroscopia de Absorção na Região do Infravermelho - FTIR}

A espectroscopia de um modo geral é todo método que utiliza radiação eletromagnética para análise da estrutura química dos compostos inorgânicos ou grupos funcionais de uma substancia orgânica. A captação de dados físico-químicos é feita através de transmissão, absorção ou reflexão de energia radiante que incide sobre a amostra (DOMINGOS et al, 2013). Ao ser excitada por uma fonte de energia, a substância pode emitir ou absorver radiação em um determinado comprimento de onda e, a partir de uma amostra submetida à análise, obtém-se dados sobre a sua estrutura, bem como natureza das ligações, comprimento das ligações, geometria das ligações etc. (SANTOS, 2010).

Nesse experimento foi usada a espectroscopia de absorção a partir da medição do comprimento de onda absorvido pela molécula, quando convertido em energia vibracional. As diferentes formas de vibração da molécula dão origem ao espectro vibracional do qual as bandas são características dos grupos funcionais da molécula (JEFFERY et al., 1992 apud SANTIAGO, 2009).

Os espectros de absorção na região do infravermelho foram obtidos em um espectrofotômetro de infravermelho por transformada de Fourier da Bomem, modelo MB 102, usando brometo de potássio $(\mathrm{KBr})$ como agente dispersante. As pastilhas foram preparadas pela mistura de aproximadamente $0,7 \mathrm{mg}$ de amostra com uma quantidade suficiente de $\mathrm{KBr}$ para se atingir a concentração de $1 \%$ em massa da amostra. Em seguida, a mistura foi homogeneizada em um almofariz, transferida para o empastilhador e submetida a uma pressão de 8,0 ton. $\mathrm{cm}^{-2}$, formando uma pastilha fina e translúcida. Os espectros foram obtidos na região de 4000 a 400 $\mathrm{cm}^{-1}$. A caracterização foi realizada no Laboratório de Análise Térmica e Materiais da Universidade Federal do Rio Grande do Norte

\section{RESULTADOS}

A espectroscopia de absorção na região do infravermelho é de grande importância no que diz respeito ao estudo dos grupos funcionais existentes e ao tipo de ligação na estrutura, já que muitos funcionam como sitio ativos para os compostos a serem adsorvidos (OLIVEIRA, 2001), além de facilitar o entendimento da estrutura do material analisado.

O gráfico 01 apresenta o espectro de infravermelho da vermiculita expandida, no qual observa-se uma banda de absorção larga e intensa na região de $3408 \mathrm{~cm}^{-1}$ referente a vibrações de deformação axiais de O-H livres dos grupos (SiO-H) da estrutura (SILVERSTEIN et al, 2002). Outra banda de intensidade média pode ser vista em $1652 \mathrm{~cm}^{-1}$, atribuída à deformação angular simétrica da ligação $\mathrm{O}-\mathrm{H}$. A existência de uma banda de intensidade forte na região de $1002 \mathrm{~cm}^{-1}$ corresponde à região de estiramento assimétrico de Si-O-Si e Si-OAl. A banda de intensidade média em $453 \mathrm{~cm}^{-1}$ está relacionada à vibração de deformação Si-O (LUCAS, 2013; DAMAZIO et al, 2014). 


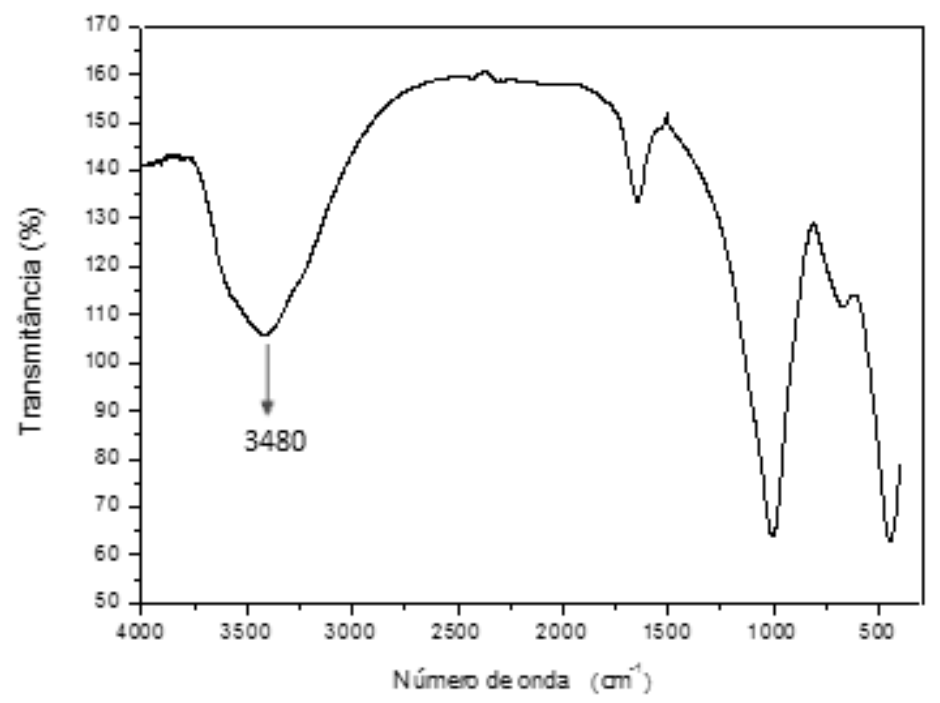

Gráfico 01. Espectro de FTIR da amostra de vermiculita expandida.

O gráfico 02 apresenta o espectro de infravermelho da turfa. Observa-se banda larga e intensa centrada na região de $3443 \mathrm{~cm}^{-1}$, atribuída ao estiramento $\mathrm{O}-\mathrm{H}$ de água e argilas. A região da banda em $2928 \mathrm{~cm}^{-1}$ é atribuída ao estiramento antissimétrico e simétrico de C-H alifático. A presença de anéis aromáticos pode ser verificada devido ao estiramento $\mathrm{C}$-H de alquenos e/ou aromáticos na banda da região de $1591 \mathrm{~cm}^{-1}$ referente ao estiramento $\mathrm{C}=\mathrm{C}$ de alquenos e/ou aromáticos. Pode-se observar estiramento C-O de álcoois e/ou fenóis e $\mathrm{SiO}$ na região de $1017 \mathrm{~cm}^{-1}$, indicando a presença de álcoois e impurezas de silicatos (FUKAMACHI, 2007).

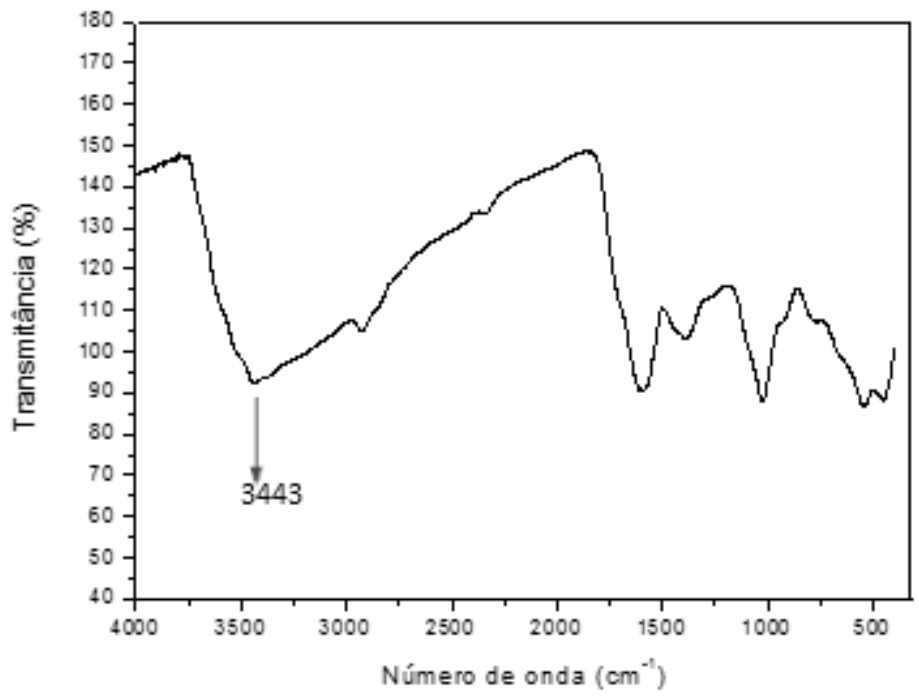

Gráfico 02. Espectro de FTIR da amostra de turfa natural.

O gráfico 03 apresenta o espectro de infravermelho da cera de carnaúba. Observa-se no espectro uma banda larga centralizada em $3442 \mathrm{~cm}^{-1}$ que revela a presença da ligação $\mathrm{O}-\mathrm{H}$ de ácidos carboxílicos. As absorções em 2920, $2852 \mathrm{~cm}^{-1}$ são referentes ao estiramento da ligação C-H. O pico $1729 \mathrm{~cm}^{-1}$ é referente ao estiramento da ligação $\mathrm{C}=\mathrm{O}$, um sinal em $1464 \mathrm{~cm}^{-1}$ de deformação angular simétrica no plano $\mathrm{CH}_{2}$ e um sinal em $1172 \mathrm{~cm}^{-1}$ de estiramento da ligação C-O e uma outra banda em $717 \mathrm{~cm}^{-1}$ que indica absorção de C-H angular fora do plano. Os demais picos presentes apresentam baixa intensidade. 


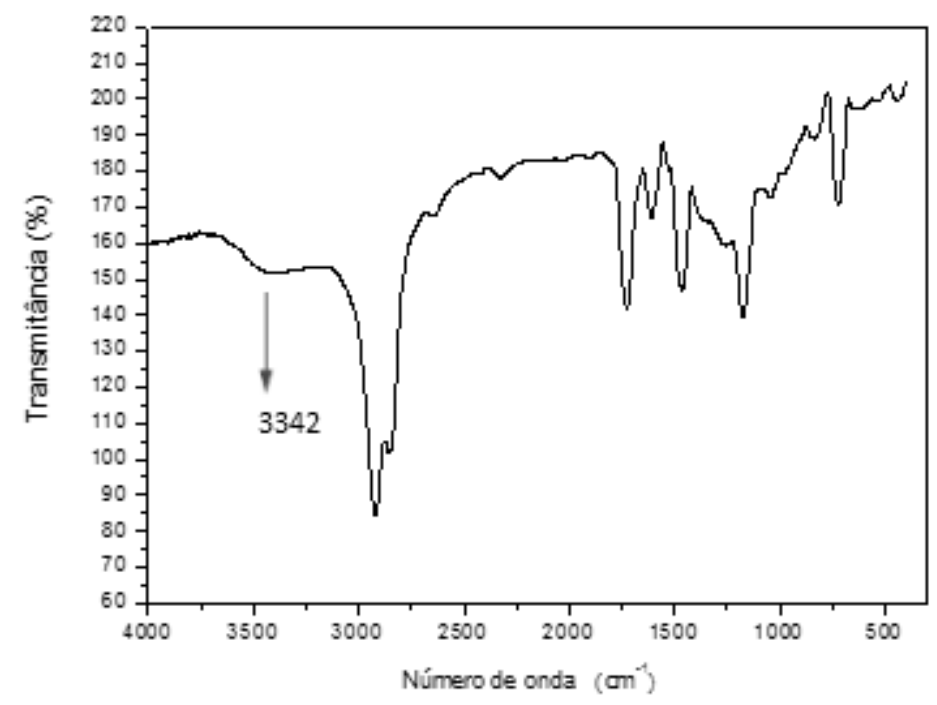

Gráfico 03. Espectro de FTIR da amostra de cera de carnaúba.

O gráfico 04 corresponde ao espectro de infravermelho da amostra de vermiculita hidrofobizada com $20 \%$ de cera de carnaúba. Pode-se observar o aparecimento da banda de absorção característica da cera de carnaúba em $2919 \mathrm{~cm}^{-1}$ correspondente ao estiramento da ligação C-H. O pico em $1454 \mathrm{~cm}^{-1}$ corresponde ao dobramento da ligação C-H (CARVALHO; FIGUEIREDO, 2013). A banda característica de grupamentos $\mathrm{OH}$ da cera não pode ser identificada já que nas mesmas regiões do espectro aparece a mesma vibração de $\mathrm{OH}$ da vermiculita. Com isso fica evidenciada a incorporação da cera de carnaúba da vermiculita (LUCAS, 2013).

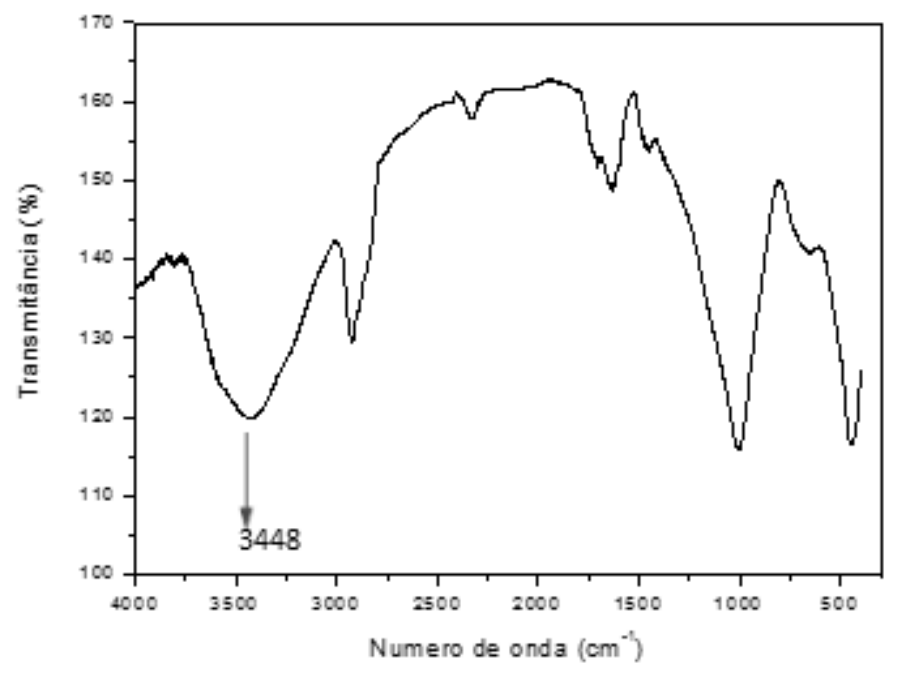

Gráfico 04. Espectro de FTIR da amostra de vermiculita hidrofobizada.

A espectroscopia na região do infravermelho da turfa hidrofobizada com $20 \%$ de cera de carnaúba (Gráfico 05) mostra uma banda larga de absorção na região de $3453 \mathrm{~cm}^{-1}$, atribuída ao estiramento O-H. A região de banda em $2913 \mathrm{~cm}^{-1}$ representa um estiramento antissimétrico e simétrico de C-H alifático. A absorção em $1706 \mathrm{~cm}^{-1}$ com pequeno pico é referente ao estiramento da ligação $\mathrm{C}=\mathrm{O}$ causado pela presença da cera de carnaúba. Pode-se observar na região da banda $1027 \mathrm{~cm}^{-1}$ um característico estiramento de Si-O. 


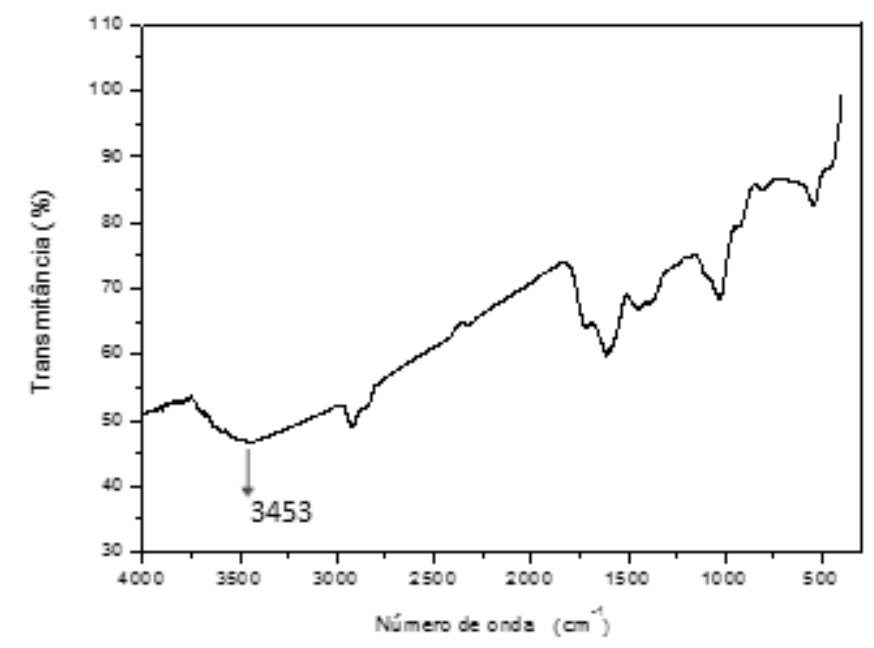

Gráfico 05. Espectro de FTIR da amostra de turfa hidrofobizada

\title{
4. CONCLUSÃO
}

Os resultados da caracterização da turfa e da vermiculita, confirmam a presença de grupos funcionais orgânicos nos adsorventes, esses grupos funcionais fazem da vermiculita e turfa um adsorvente potencial para remoção de contaminantes através da adsorção química e/ou física. $\mathrm{O}$ espectro das amostras hidrofobizadas apresentaram os sinais característicos de algumas transições vibracionais dos componentes da cera de carnaúba, mostrando a presença do agente hidrofobizante nos materiais adsorventes.

\section{Characterization of peat and vermiculite hydrophobized by Absorption Spectroscopy in Region Infravermelho- FTIR}

\begin{abstract}
:
Natural materials such as minerals and clay minerals have been investigated for alternative use in environmental areas for the purpose of solving many problems that impact the environment. The peat and vermiculite are gaining attention today because of the power that adsorb impurities present in water and wastewater contaminated with hydrocarbons and metals, and other toxic substances. Peat is classified as an organic-mineral originated from the decomposition of vegetable little development in freshwater environment. It is a polar and highly porous material. Vermiculite is a mineral derived from igneous rocks, occurs in basic rocks in the alkaline rocks and sometimes in pegmatites and usually forms the weathering suffered by biotite. Several methods have been the subject of studies aimed at activating or ning the ability of these materials to be used as adsorbents of pollutants, particularly hydrophobic, that is to make an adsorbent material with aversion to water and improve the adsorption capacity of the same. Countless studies attest to carnauba wax efficient hydrophobic process of clay materials, making the same affinity to organic compounds. This study aims to characterize the peat and vermiculite in natural and hydrofobized form with carnauba wax by absorbing technique of the infrared Fourier transform, to better understand the properties of these materials. The results
\end{abstract}


obtained in this study confirmed the heterogeneity of the studied materials as well, confirmed the adherence of carnauba wax used in the hydrophobic process to them.

Keywords: Clay - minerals; adsorbent ; vermiculite ; Hydrophobic .

\section{REFERÊNCIAS}

BORBA, L. L. S.; OLIVEIRA, M. F. D.; MELO, M. A. F.; MELO, D. M. A.; PERGHER, S. B. C. Preparação de adsorventes à base de materiais naturais hidrofobizados com cera de carnaúba. Perspectiva, Erechim, v. 37, n. 139, p.37-46, set. 2013.

CARVALHO, D. G.; FIGUEIREDO, A. B. da S. Síntese e caracterização de nanopartículas de $\mathrm{NiFe} 2 \mathrm{O} 4$ utilizando o método de sol-gel/combustão e combustão homogênea. Matéria (Rio J.). 2013, vol.18, n.2, pp. 1323-1328.

COElHO, A. C. V.; SANTOS, P. de S.; SANTOS, H. de S. Argilas especiais: o que são, caracterização e propriedades. Quim. Nova, v. 30, n. 1, p. 146-152, 2007

CURBELO, F. D. da S. Estudo da remoção de óleo em águas produzidas na industria de petróleo, por adsorção em coluna utilizando a vermiculita expandida e hidrofobizada. Dissertação de Mestrado - Programa de Pós-graduação em Engenharia Química - Departamento de Engenharia Química, Universidade Federal do Rio Grande do Norte, Natal, 2002.

DAMAZIO, D.; DUTRA, R. de C. L.; DINIZ, M. F; MATTOS, E. da C. Determinação por FTIR de transmissão e reflexão (UATR) de etileno e propileno em EPDM. Polímeros. 2014, vol.24, n.6, pp. 703-710

DOMINGOS, E.; PEREIRA, T. M. C.; CASTRO, E. V. R. de; ROMÃO, W; SENA, G. L. de S.; GUIMARÃES, R. C. L. Monitorando a degradação da poliamida 11 (PA-11) via espectroscopia na região do infravermelho médio com transformada de fourier (FTIR). Polímeros. 2013, vol.23, n.1, pp. 37-41. Epub Oct 30, 2012

FRANCHI, J.G. Aplicação de turfa na recuperação de solos degradados pela mineração de areia. Dissertação (Mestrado em Engenharia Mineral) - Escola Politécnica da Universidade de São Paulo. 105f. 2000.

FRANCHI, J. G. A utilização de turfa como adsorvente de metais pesados: $\mathrm{O}$ exemplo da contaminação da bacia do rio Ribeira de Iguape por chumbo e metais associados. 2004. $178 \mathrm{f}$. Tese- Curso de Programa de Pós Graduação em Geoquímica e Geotectônica, Instituto de Geociências, Universidade de são Paulo, São Paulo, 2004.

FRANCHI, J. G., SÍGOLO, J. B., LIMA, J. R. B. Turfa utilizada na recuperação ambiental de áreas mineradas - metodologia para avaliação laboratorial. Revista Brasileira de Geociências, 255-262, 2003.

FRANCHI, J. G., MOTTA, J. F. M,. UOSUKAINEN, H., SÍGOLO, J. B. Peat in Brazil: geology, reserves, production and use. In: INTERNATIONAL PEAT CONGRESS, 12. 2004, Tampere. Proceedings... Saarijävi, IPS. 2004. p. 627-632.

FUKAMACHI, C. R. B. Fertilizantes de liberação lenta de nitrogênio por nitração de turfa e xisto e por intercalação de uréia em argilominerais do grupo do caulim. 2007. 116 f. Tese (Doutorado) - Curso de Programa de Pós - Graduação em Química, Setor de Ciências Exatas da Universidade Federal do Paraná., Universidade Federal do Paraná., Curitiba, 2007. 
LUCAS, G. H. Hidrofobização, caracterização e aplicação da vermiculita para remoção de óleo insolúvel em água. 2013. 74 f. Dissertação (Mestrado) - Curso de Programa de Pósgraduação em Química, Centro de Ciências Exatas e da Terra. Universidade Federal do Rio Grande do Norte, Natal, 2013.

MARTINS, J.; Vermiculita é transformada em mineral hidrofóbico, por Mara Figueira, Ciência Hoje, Jornal do Brasil, Rio de Janeiro - RJ, 2000.

OLIVEIRA, L. F. C. de. Espectroscopia Molecular. Cadernos Temáticos de Química Nova na Escola, n. 4, p. 24-30, 2001.

SANTIAGO, R. C. Rejeito de xisto como adsorvente para remoção de fenol em águas produzidas na indústria de petróleo. 2009. 100 f. Dissertação (Mestrado) - Curso de Pósgraduação em Ciência e Engenharia de Petróleo, Centro de Ciências Exatas e da Terra, Universidade Federal do Rio Grande do Norte, Natal, 2009.

SANTOS, A. da S. Aplicação da parafina e do óleo de linhaça como hidrofobizantes da vermiculita, para remoção de derivados de óleo diesel presentes em água produzida sintética. 2010. 120 f. Dissertação (Mestrado) - Curso de Programa de Pós-graduação em Química, Centro de Ciências Exatas e da Terra, Universidade Federal do Rio Grande do Norte, Natal, 2010.

SILVERSTEIN, R. M.; BANLER, G. C.; MORRILL, T. C. Identificação Espectrométrica de Compostos Orgânicos. Guanabara Koogan, 6a . Edição, Cap. 3, p. 85, Rio de Janeiro, 2002.

UGARTE, J. F. de O.; SAMPAIO, J. A.; FRANÇA, S. C. A.. Vermiculita. In: LUZ, Adão Benvindo da; LINS, Fernando Antonio Freitas. Rochas \& minerais industriais: Usos e especificações. Rio de Janeiro: Cetem/mct, 2005. Cap. 32. p. 677-698. 